\title{
Efectividad simbiótica de dos cepas de Rhizobium $s p$. en cuatro variedades de frijol (Phaseolus vulgaris L.) en Perú
}

\author{
Symbiotic effectiveness of two Rhizobium sp. strains in four \\ common bean (Phaseolus vulgaris L.) varieties in Perú \\ Héctor Cantaro-Segura ${ }^{*}$, Amelia Huaringa-Joaquín², Doris Zúñiga-Dávil ${ }^{1}$
}

\begin{abstract}
RESUMEN
Las leguminosas son un amplio grupo de plantas de gran importancia en todo el mundo, que tienen la capacidad capaces de entrar en simbiosis con rizobios que fijan el nitrógeno atmosférico y lo convierten en formas orgánicas disponibles para su uso. La efectividad simbiótica de dos cepas de Rhizobium sp. (LMT10 y LMT15), una cepa nativa y una fertilización nitrogenada de $100 \mathrm{~kg}$ $\mathrm{N} \mathrm{ha}^{-1}$ fue evaluada en cuatro variedades de frijol (Canario Centenario, Canario CIFAC, Blanco Molinero y Rojo Molinero). Las variedades con el rendimiento más alto fueron Blanco Molinero con $2.636 \mathrm{~kg} \mathrm{ha}^{-1}$ y Canario Centenario con $2.523 \mathrm{~kg} \mathrm{ha}^{-1}$. Se encontró una interacción significativa entre fuente de nitrógeno y variedad para número de vainas por planta $(\mathrm{p}<0,05)$ e índice de cosecha $(\mathrm{p}<0,01)$, donde el mejor tratamiento fue Canario Centenario con fertilización nitrogenada $\left(3.062 \mathrm{~kg} \mathrm{a}^{-1}\right), 21,4$ vainas por planta y $65 \%$ de índice de cosecha. Aunque no hubo diferencias significativas entre las fuentes de nitrógeno, las cepas inoculadas mostraron una alta efectividad simbiótica y una especificidad de las cepas aisladas de Blanco Molinero, lo que se reflejó en los parámetros morfofisiológicos y de rendimiento. Los tratamientos inoculados alcanzaron el $90 \%$ de los rendimientos obtenidos con los fertilizantes nitrogenados, demostrando su uso potencial en la agricultura sostenible al disminuir la polución derivada del empleo de fertilizantes sintéticos en el ecosistema, además de ser una alternativa de bajo costo para los agricultores en el mundo.

Palabras clave: Rhizobium, efectividad, simbiosis, frijol, variedades.
\end{abstract}

\begin{abstract}
Legumes are a large group of important plants worldwide that can enter in symbiosis with a bacteria called rhizobia, which settle the atmospheric nitrogen converting it into available organic forms for plant use. The symbiotic effectiveness of two Rhizobium sp. strains LMT 10 and LMT15, an indigenous soil strain, and an $\mathrm{N}$-fertilization rate of $100 \mathrm{~kg} \mathrm{~N}$. ha-l (nitrogen sources) were evaluated on four commercial common bean varieties (Canario Centenario, Canario CIFAC, Blanco Molinero, and Rojo Molinero). The varieties with the highest yields were Blanco Molinero $\left(2,636 \mathrm{~kg} \mathrm{ha}^{-1}\right)$ and Canario Centenario $\left(2523 \mathrm{~kg}^{\text {. h }}^{-1}\right)$. A significant interaction of bean variety by nitrogen source was found for pod number per plant $(p<0.05)$ and harvest index $(p<0.01)$, where the best treatment was Canario Centenario with $\mathrm{N}$-fertilization (3,062 $\left.\mathrm{kg} \mathrm{ha}^{-1}\right), 21.44$ pods per plant and $64.97 \%$ harvest index respectively. Although there were no statistical differences between the nitrogen sources, the strains showed high symbiotic effectiveness and high specificity of the strains. The inoculated treatments reached $90 \%$ of the yields obtained with the nitrogen fertilizers revealing their potential use in sustainable agriculture.
\end{abstract}

Keywords: Rhizobium, effectiveness, symbiosis, common bean, varieties.

\section{Introducción}

El frijol común es la leguminosa de grano más importante del mundo (Rahmani et al., 2011) ya que representa el $87 \%$ de las leguminosas consumidas a nivel mundial (Pedrosa et al. 2015). En Latinoamérica, y especialmente en
Perú, este cultivo es parte principal de la dieta diaria de personas pobres, donde tiene la más alta producción y consumo debido a que reúne buenas cualidades agronómicas (precocidad, buen potencial de rendimiento, buena adaptación para la costa y valles interandinos cálidos, utilidad en programas de rotación, mejorador de suelos

1 Departamento de Fitotecnia, Facultad de Agronomía. Universidad Nacional Agraria La Molina, Apartado Postal 456. Lima, Perú.

2 Laboratorio de Ecología Microbiana y Biotecnología "Marino Tabusso", Universidad Nacional Agraria La Molina, Apartado Postal 456. Lima 1, Perú.

* Corresponding author: hector.cs257@gmail.com; hcantaro@lamolina.edu.pe

Fecha de Recepción: 24 Mayo, 2019.

Fecha de Aceptación: 8 Agosto, 2019. 
por su propiedad de fijar nitrógeno mediante la simbiosis con Rhizobium) y nutricionales (22-28\% de proteínas, 59-60\% de carbohidratos, vitaminas, minerales y fibras solubles). Constituye la mayor fuente de proteínas (25-35\% del consumo proteínico mundial), por encima de las fuentes animales ( $70 \%$ de proteínas en la dieta) [Broughton et al., 2003; Sánchez et al., 2014]. El Perú tiene condiciones agroecológicas favorables que incrementan la producción del frijol significativamente, tanto para satisfacer el consumo nacional como para disponer de una mayor oferta exportable (Camarena et al., 2009). Además, ese país presenta un gran potencial genético en este cultivo debido a que es uno de sus centros de origen (Kwak y Gepts, 2009), y pertenece al acervo génico andino (Okii et al., 2014).

Uno de los principales elementos en la agricultura sustentable es el manejo efectivo del nitrógeno $(\mathrm{N})$ en el medioambiente (Broughton et al., 2003). Para obtener un buen rendimiento en los cultivos agrícolas es necesario utilizar fertilizantes químicos nitrogenados, los cuales elevan los costos de producción, lo que afecta la economía del productor y del consumidor (Erisman, 2011). Además, la fertilización mineral con $\mathrm{N}$ involucra muchos problemas, como un consumo energético excesivo debido al proceso de síntesis y contaminación del agua con nitratos, como consecuencia de la lixiviación (Nótás et al., 2007), con efectos negativos en los ecosistemas y la salud humana (Mulas et al., 2011). Una de las alternativas para resolver este problema de manera ecológica (Lichtfouse et al., 2008) es la utilización de inoculantes elaborados con bacterias fijadoras de nitrógeno presentes en el suelo e inductoras de nódulos, comúnmente conocidas como rizobios. Estas bacterias, al establecer una relación simbiótica con las leguminosas, tienen la capacidad de fijar nitrógeno atmosférico y convertirlo en nitrógeno orgánico, el cual puede ser utilizado por la planta, e incorporarlo en sus procesos metabólicos posteriores (Alberton et al., 2006).

Las variedades de frijol exhiben una fuerte dependencia de los fertilizantes $\mathrm{N}$ para completar un buen rendimiento en el campo debido a su considerable variabilidad en su capacidad para nodular y fijar N2. Por ello es considerado un pobre fijador de nitrógeno comparado con otras leguminosas (Rahmani et al., 2011), y esto ha llevado a una pérdida de interés en la investigación sobre la simbiosis frijol-Rhizobium en los últimos años. $P$. vulgaris puede entrar en simbiosis con bacterias del género Rhizobium y Sinorhizobium (Mulas et al., 2011) y el establecimiento de una simbiosis efectiva depende de muchos factores ambientales y prácticas de manejo agronómico (Unkovich et al., 2008). Un factor crucial en el éxito de esta interacción es la especificidad de las cepas bacterianas con su hospedante, donde la ausencia de un suficiente número de rizobios efectivos y compatibles constituye uno de los elementos limitantes en la fijación de nitrógeno (Rodríguez-Navarro et al., 2000). Aunque algunos autores han encontrado una respuesta ligera a la inoculación de cepas no nativas (Mathu et al., 2012; Ndungu et al., 2018), otros han observado que una inoculación efectiva con cepas nativas o la presencia de cepas nativas en el suelo producen rendimientos de grano similares a los obtenidos con una fertilización nitrogenada (Mulas et al., 2011; Sánchez et al., 2014), al igual que la inoculación con otros organismos como Pseudomonas o rizobacterias promotoras del crecimiento vegetal (PGPR) (Sánchez et al., 2014).

Los objetivos de este estudio fueron (I) determinar la efectividad simbiótica de dos cepas de Rhizobium sp., LMT10 y LMT15, en cuatro variedades de frijol; (II) establecer el efecto de la fijación biológica del nitrógeno en el desarrollo y el rendimiento de grano seco de cuatro variedades de frijol y (III) comparar el efecto de la fertilización nitrogenada en la simbiosis y el rendimiento en las cuatro variedades de frijol.

\section{Materiales y métodos}

\section{Material vegetal}

Las variedades de frijol común usadas en este experimento fueron cuatro variedades comerciales difundidas y de amplia aceptación, proporcionadas por el Programa de Investigación y Proyección Social de Leguminosas de Granos de la Universidad Nacional Agraria La Molina. Las variedades son Canario Centenario (CCENT), Canario CIFAC (CCIF), Blanco Molinero (BM) y Rojo Molinero (RM). Las dos primeras variedades son frijoles de grano amarillo, BM es blanco y RM rojo. 
Tabla 1. Caracterización fenotípica y simbiótica in vitro de las cepas de Rhizobium usadas en este experimento.

\begin{tabular}{cccccc}
\hline Cepa & NN & PFT $(\mathrm{g})$ & PST $(\mathrm{g})$ & $\mathrm{pH}$ & Temp $\left({ }^{\circ} \mathrm{C}\right)$ \\
\hline LMT10 & 28 & 2,65 & 0,29 & $4-8,8$ & $28-37$ \\
LMT15 & 14 & 2,65 & 0,31 & $4-8,8$ & $28-37$ \\
\hline
\end{tabular}

NN: número de nódulos; PFT: Peso fresco de tallos, PST: Peso seco de tallos.

Fuente: Laboratorio de Ecología Microbiana y Biotecnología Marino Tabusso.

\section{Cepas bacterianas}

Como inoculantes, se utilizaron las cepas LMT10 y LMT15, de Rhizobium sp. (Tabla 1), aisladas de la variedad Blanco Molinero, y pertenecientes a la colección del Laboratorio de Ecología Microbiana y Biotecnología Marino Tabusso de la Universidad Nacional Agraria La Molina (UNALM).

\section{Análisis del suelo}

En el campo experimental, se tomaron al menos quince muestras a una profundidad de $20 \mathrm{~cm}$. Luego se mezcló y se extrajo $1 \mathrm{~kg}$ para su análisis físico y químico en el laboratorio. El análisis de suelo fue realizado de acuerdo a los lineamientos establecidos por el Soil Survey Staff (2014).

\section{Condiciones meteorológicas}

Según el sistema modificado de Köppen, basado en promedios anuales de precipitación y promedios anuales de temperatura, a La Molina le corresponde la clasificación de desierto subtropical

Tabla 2. Propiedades físicas y químicas del suelo en el sitio experimental.

\begin{tabular}{lcl}
\hline \multicolumn{1}{c}{ Análisis } & Resultado & \multicolumn{1}{c}{ Método } \\
\hline $\mathrm{pH}$ & 7,92 & Suelo: Agua Relación $1: 1$ \\
$\mathrm{CE}(1: 1) \mathrm{dS} / \mathrm{m}$ & 0,35 & Suelo: Agua Relación 1:1 \\
Materia Orgánica $(\%)$ & 1,07 & Walkley y Black \\
Fósforo (ppm) & 10,7 & Método del Olsen modificado \\
Potasio (ppm) & 176 & Extracción con acetato de amonio \\
Clase textural & Franco & Método del hidrómetro \\
\hline
\end{tabular}

Fuente: Laboratorio de Análisis de Suelos, Plantas, Agua y Fertilizantes. UNALM. árido caluroso. Durante el ciclo del cultivo, la precipitación, humedad y temperatura media se determinó en el sitio experimental (Tabla 3).

\section{Diseño experimental}

El diseño experimental empleado en el campo experimental de la UNALM (1204'57'S76 56'49' W) fue el de Diseño de Bloques Completos al Azar con Arreglo Factorial 4 x 4 (cuatro variedades, cuatro fuentes de N) y tres bloques, 16 tratamientos y 48 unidades experimentales o parcelas en total. Las parcelas fueron de $9,6 \mathrm{~m}^{2}$, con tres hileras distanciadas $0,8 \mathrm{~m}$ entre ellas y $0,3 \mathrm{~m}$ entre plantas, con un total de 66 plantas por unidad. En todas las unidades experimentales se aplicaron $100 \mathrm{~kg} \mathrm{ha}^{-1}$ de PK granulado en la hilera inmediatamente después de ser sembrado, cantidad que fue calculada con base en el análisis de suelo y los rendimientos esperados. Los tratamientos fueron divididos en frijoles inoculados con dos cepas de Rhizobium, más los dos tratamientos no inoculados, uno no fertilizado con $\mathrm{N}$ y otro fertilizado con $100 \mathrm{~kg} \mathrm{~N} \mathrm{ha}^{-1}$ en forma de nitrato de amonio. Adicionalmente, se aplicaron ocho sacos de compost al campo antes de la siembra. El herbicida Linuron fue utilizado en preemergencia para controlar las malezas a una dosis de $21 \mathrm{ha}^{-1}$. El riego se aplicaba cuando era necesario, aunque se presentó un pequeño estrés por sequía que luego fue subsanado. El bioestimulante Agrostemin fue colocado a dosis de $1 \mathrm{ha}^{-1}$. Después, en el transcurso del cultivo, se aplicaron micronutrientes como Oligomix Co, debido a que el cobalto es esencial en el desarrollo y función del nódulo. Las plagas $y$ enfermedades fueron manejadas bajo el enfoque

Tabla 3. Parámetros climatológicos de la zona de La Molina en el periodo experimental.

\begin{tabular}{lccc}
\hline Mes & $\begin{array}{c}\mathrm{T}^{\mathrm{o}} \text { Media } \\
\left({ }^{\circ} \mathrm{C}\right)\end{array}$ & $\begin{array}{c}\text { Humedad Relativa } \\
(\%)\end{array}$ & $\begin{array}{c}\text { Precipitación } \\
(\mathrm{mm})\end{array}$ \\
\hline Julio & 15,08 & 85,09 & 3,90 \\
\hline Agosto & 15,03 & 84,15 & 6,80 \\
Septiembre & 16,00 & 82,74 & 3,60 \\
Octubre & 17,47 & 73,87 & 0,00 \\
Noviembre & 18,97 & 76,89 & 0,60 \\
\hline
\end{tabular}

Fuente: Estación Meteorológica Alexander von Humboldt La Molina. 
de manejo integrado de plagas. La cosecha se llevó a cabo 140 días después de la siembra.

\section{Variables evaluadas}

Para el muestreo de evaluación fueron cosechadas manualmente diez plantas al azar de cada unidad experimental. El muestreo se realizó dos veces: I) al comienzo de la fase reproductiva aproximadamente a los 45 días después de la plantación (DDP) y II) en la cosecha a los 140 días después de la plantación (DAP). En el primer muestreo se evaluó número (NN), tamaño (TN) y peso fresco de nódulos (PFN). Los nódulos fueron subsecuentemente secados por 48 horas a $65^{\circ} \mathrm{C}$ para determinar el peso seco de nódulos (PSN). De la misma manera, el peso seco de raíces (PSR) y el de follaje (PSF) se midieron después de secar en una estufa por 72 horas a $65^{\circ} \mathrm{C}$.

La cosecha se realizó cuando las semillas y las plantas estaban secas, con una ligera variación entre días en los genotipos. Las plantas fueron removidas completamente y se contó el número de plantas por parcela. Las semillas fueron secadas al sol por 2 días y se determinó el peso seco de semillas por parcela. El rendimiento para cada parcela fue calculado como se indica: el peso seco de semillas por parcela dividido por el número de plantas cosechadas en esa parcela, multiplicado por el número de plantas calculadas por hectárea. Las plantas muestreadas en las parcelas se usaronposteriormente para: I) determinación del número de vainas (NVP) y el número de granos por vaina (NGV), II) medición del peso fresco y seco de las plantas. También, los días a floración (DAF) y la altura de planta (AP) fueron calculados en los parámetros morfofisiológicos. En los parámetros de nodulación se incluyeron parámetros cualitativos como color y forma de nódulos. Finalmente en los parámetros de rendimiento se midió el peso seco de cien semillas (PCS) y el índice de cosecha (IC).

\section{Análisis estadístico}

Los datos del experimento fueron sometidos primero a test de normalidad y homogeneidad de varianza para cada variable y luego al análisis de varianza (ANOVA) usando un nivel de significancia de $\mathrm{p}=0,05$. Las medias de los tratamientos fueron comparadas utilizando Duncan como prueba post hoc de significancia (SAS 9,4 para Windows).

\section{Resultados}

\section{Análisis del suelo y condiciones meteorológicas}

El suelo donde se realizó el trabajo experimental es profundo, de buen drenaje, permeabilidad moderada, textura media, estructura granular media y moderada, de consistencia friable en húmedo. En cuanto a las propiedades químicas, el suelo mostró un bajo contenido de sales, $\mathrm{pH}$ medianamente alcalino, bajo contenido de materia orgánica y concentraciones medias de $\mathrm{P}$ y $\mathrm{K}$ en óptimos niveles. En las relaciones entre los cationes involucrados se observa una predominancia del $\mathrm{Ca}$ y el Mg $(94,19 \%$ PSB) y una relación Ca/Mg de 6,53 y una relación $\mathrm{K} / \mathrm{Mg}$ de 0,4 . Estos resultados se presentan en la Tabla 1. La Tabla 2 muestra un incremento marcado de la temperatura en octubre y noviembre debido a la estación primaveral, la precipitación también disminuye al mismo ritmo del cambio de estación de invierno a primavera, y una tendencia similar en la humedad relativa (\%).

\section{Efectos de Rhizobium y el N sobre los parámetros morfofisiológicos}

Las variables morfofisiológicas fueron determinados bajo condiciones de campo. Los días a floración se evaluaron en plena floración y los valores están comprendidos entre 45,35 y 55,35 días, en Canario Centenario y Blanco Molinero, respectivamente. Como se muestra en la Tabla 4, la variedad CIFAC tuvo los mayores valores en altura, peso fresco de follaje (PFF), peso seco de follaje y peso seco de raíces; y la variedad Rojo Molinero el menor valor en esas variables. Con respecto a las fuentes de N, la cepa LMT10 destacó para altura, peso fresco de follaje, peso fresco de raíces y peso seco de follaje, y los menores valores los obtuvo el tratamiento testigo (-N), donde solo intervinieron las cepas nativas.

El análisis de varianza indicó que hubo diferencias extremadamente significativas $(\mathrm{p}<0,001)$ entre las variedades para las variables dependientes DAF, AP, PFF, PSF y PSR; y altamente significativas para PFR $(p<0,01)$. Respecto a fuentes de N, el análisis de varianza no mostró diferencias significativas para ninguna de las variables dependientes. Además, no hubo significancia estadística para la interacción entre 
Tabla 4. Resultados promedios de las variables morfofisiológicas en el experimento.

\begin{tabular}{|c|c|c|c|c|c|c|}
\hline Efecto principal & DAF (días) & ALT (cm) & PFF (g) & PFR (g) & PSF (g) & PSR (g) \\
\hline Variedad & $* * *$ & $* * *$ & $* * *$ & $* *$ & $* * *$ & $* * *$ \\
\hline CCENT & $46,7^{\mathrm{b}} \pm 0,83$ & $38,65^{\mathrm{ab}} \pm 2,46$ & $36,75^{\mathrm{a}} \pm 4,84$ & $2,61^{\mathrm{a}} \pm 0,30$ & $9,08^{a} \pm 1,28$ & $2,43^{a} \pm 0,27$ \\
\hline CCIF & $50,9^{a} \pm 0,71$ & $44,47^{a} \pm 2,01$ & $40,81^{\mathrm{a}} \pm 5,29$ & $2,58^{a} \pm 0,06$ & $10,45^{\mathrm{a}} \pm 0,79$ & $2,11^{\mathrm{a}} \pm 0,12$ \\
\hline BM & $53,4^{\mathrm{a}} \pm 1,00$ & $34,08^{b} \pm 2,62$ & $40,34^{\mathrm{a}} \pm 5,36$ & $1,53^{b} \pm 0,19$ & $8,51^{\mathrm{a}} \pm 1,01$ & $0,41^{b} \pm 0,03$ \\
\hline $\mathrm{RM}$ & $48,8^{\mathrm{b}} \pm 0,63$ & $22,99^{c} \pm 1,26$ & $11,20^{\mathrm{b}} \pm 0,97$ & $1,08^{\mathrm{b}} \pm 0,10$ & $2,15^{\mathrm{b}} \pm 0,20$ & $0,42^{b} \pm 0,06$ \\
\hline Fuente de $\mathrm{N}$ & Ns & ns & $\mathrm{ns}$ & ns & ns & ns \\
\hline Control (-N) & $48,5^{\mathrm{a}} \pm 0,92$ & $33,55^{\mathrm{a}} \pm 2,69$ & $23,82^{\mathrm{b}} \pm 3,87$ & $1,60^{a} \pm 0,22$ & $5,90^{\mathrm{a}} \pm 0,96$ & $1,43^{a} \pm 0,23$ \\
\hline LMT10 & $51,0^{\mathrm{a}} \pm 1,16$ & $37,52^{\mathrm{a}} \pm 3,35$ & $39,76^{a} \pm 6,58$ & $1,71^{\mathrm{a}} \pm 0,26$ & $8,73^{\mathrm{a}} \pm 1,48$ & $1,49^{a} \pm 0,27$ \\
\hline LMT15 & $48,3^{a} \pm 0,62$ & $33,21^{\mathrm{a}} \pm 2,79$ & $30,13^{\mathrm{ab}} \pm 4,84$ & $1,64^{\mathrm{a}} \pm 0,33$ & $7,52^{\mathrm{a}} \pm 1,26$ & $1,50^{\mathrm{a}} \pm 0,31$ \\
\hline$+\mathrm{N}$ & $51,8^{\mathrm{a}} \pm 1,01$ & $35,91^{\mathrm{a}} \pm 3,71$ & $35,40^{\mathrm{ab}} \pm 6,41$ & $1,60^{\mathrm{a}} \pm 0,27$ & $8,03^{\mathrm{a}} \pm 1,38$ & $1,40^{\mathrm{a}} \pm 0,27$ \\
\hline \multicolumn{7}{|l|}{ Interacción } \\
\hline $\mathrm{V} * \mathrm{FN}$ & Ns & ns & ns & ns & ns & ns \\
\hline
\end{tabular}

Los valores son promedios de 12 plantas \pm error estándar.

En cada columna, las medias seguidas de la misma letra no difieren estadísticamente (Duncan $\mathrm{p}<0,05$ ).

Duncan significant level: $* 0,05>\mathrm{P}>0,01 ; * * 0,01>\mathrm{P}>0,001 ; * * * \mathrm{P}<0,001$, ns not significant.

VAR: variedad, CCENT: Canario Centenario, CCIF: Canario CIFAC, BM: Blanco Molinero, RM: Rojo Molinero.

$\mathrm{N}$ : tratamiento testigo, +N: fertilización nitrogenada, +LMT10 cepa LMT10, LMT15 cepa LMT15.

DAF: días a la floración, ALT: altura, PFF: peso fresco de follaje, PFR: peso fresco de raíces, PSF: peso seco de follaje, PSR: peso seco de raíces.

factores. Aunque no se observaron diferencias significativas en la fertilización nitrogenada y los tratamientos inoculados para ningún parámetro, la cepa LMT10 tuvo los más altos valores comparados con la fertilización nitrogenada.

\section{Efectos de Rhizobium y el N sobre los parámetros de nodulación}

El análisis de varianza mostró que hubo diferencias significativas $(\mathrm{p}<0,05)$ entre las variedades para tamaño de nódulos y peso fresco de nódulos; y altamente significativas para peso seco de nódulos $(\mathrm{p}<0,01)$. Como lo reportado en la literatura, en este experimento los parámetros de nodulación en los tratamientos inoculados y el control fueron significativamente mejores comparados con los tratamientos fertilizados con nitrógeno. El número de nódulos en el tratamiento control muestra el potencial de las cepas nativas en el suelo para infectar y nodular el frijol común. Sin embargo, con los tratamientos inoculados obtenemos mejores resultados.

Los efectos negativos de la fertilización nitrogenada sobre la nodulación han sido ampliamente descritos en la introducción y nuestros resultados concuerdan con lo reportado por Abayomi et al. (2008), quienes mencionan que la aplicación de fertilizantes resulta en una disminución de la formación de nódulos, pero aún hay presencia de nódulos. No se limita por completo.

\section{Efectos de Rhizobium y el N sobre los parámetros de rendimiento}

El análisis de varianza demostró que hubo diferencias extremadamente significativas $(\mathrm{p}<0,001)$ entre las variedades para las siguientes variables dependientes: número de vainas por planta, número de granos por vaina y rendimiento grano seco. Respecto a fuentes de N, el análisis de varianza no mostró diferencias significativas para ninguna de las variables dependientes. Pero sí hubo significancia estadística para la interacción entre variedades y fuentes de $\mathrm{N}$ en las variables número de vainas por planta $(\mathrm{p}<0,05)$ e índice de cosecha $(p<0,001)$.

El mayor rendimiento de los tratamientos con las cepas y con la fertilización mineral de $\mathrm{N}$ en comparación con el control no fertilizado (Tabla 5) es el resultado de una mayor significancia estadística $(p<0,001)$ en número de vainas por planta y número de granos por vaina, lo que coincide con Mulas et al. (2015). El tamaño de las semillas y, por consiguiente, el peso de cien semillas fue similar en todos los tratamientos y por 
Tabla 5. Resultados promedios de las variables de nodulación y rendimiento en el experimento.

\begin{tabular}{|c|c|c|c|c|c|c|c|c|c|}
\hline $\begin{array}{l}\text { Efecto } \\
\text { principal }\end{array}$ & NN & $\mathrm{TN}(\mathrm{mm})$ & $\operatorname{PFN}(g)$ & $\operatorname{PSN}(g)$ & NVP & NGV & $\operatorname{PCS}(\mathrm{g})$ & IC $(\%)$ & $\begin{array}{c}\text { REND } \\
\left(\mathrm{kg} \cdot \mathrm{ha}^{-1}\right)\end{array}$ \\
\hline Variedad & Ns & $*$ & $*$ & $* *$ & $* * *$ & $* * *$ & $\mathrm{~ns}$ & ns & $* * *$ \\
\hline CCENT & $10,29^{\mathrm{ab}} \pm 2,65$ & $3,86^{\mathrm{ab}} \pm 1,15$ & $0,31^{\mathrm{ab}} \pm 0,08$ & $0,04^{b} \pm 0,010$ & $18,52^{\mathrm{ab}} \pm 0,79$ & $3,61^{\mathrm{ab}} \pm 0,12$ & $55,33^{\mathrm{a}} \pm 1,86$ & $60,22^{\mathrm{a}} \pm 1,07$ & $2523,1^{\mathrm{a}} \pm 147,6$ \\
\hline $\mathrm{CCIF}$ & $8,48^{b} \pm 2,00$ & $7,03^{\mathrm{a}} \pm 1,64$ & $0,13^{\mathrm{b}} \pm 0,03$ & $0,02^{b} \pm 0,004$ & $19,71^{\mathrm{a}} \pm 0,67$ & $3,41^{\mathrm{b}} \pm 0,12$ & $53,90^{\mathrm{a}} \pm 1,90$ & $56,32^{b} \pm 1,64$ & $2360,3^{\mathrm{a}} \pm 147,6$ \\
\hline BM & $15,53^{a} \pm 2,26$ & $2,08^{c} \pm 0,18$ & $0,46^{\mathrm{a}} \pm 0,07$ & $0,09^{\mathrm{a}} \pm 0,014$ & $17,05^{\mathrm{b}} \pm 0,95$ & $3,85^{\mathrm{a}} \pm 0,17$ & $58,93^{\mathrm{a}} \pm 1,22$ & $55,29^{\mathrm{b}} \pm 2,44$ & $2635,9^{\mathrm{a}} \pm 149,9$ \\
\hline RM & $8,16^{\mathrm{b}} \pm 1,62$ & $3,94^{\mathrm{ab}} \pm 0,78$ & $0,25^{\mathrm{b}} \pm 0,06$ & $0,04^{b} \pm 0,011$ & $5,89^{c} \pm 0,49$ & $2,36^{\mathrm{c}} \pm 0,13$ & $54,13^{\mathrm{a}} \pm 1,90$ & $57,18^{\mathrm{ab}} \pm 2,13$ & $504,7^{b} \pm 43,4$ \\
\hline Fuente de $\mathrm{N}$ & Ns & ns & $\mathrm{ns}$ & $\mathrm{ns}$ & ns & ns & ns & ns & ns \\
\hline Control & $11,39^{\mathrm{a}} \pm 2,13$ & $5,05^{\mathrm{a}} \pm 1,31$ & $0,30^{\mathrm{a}} \pm 0,08$ & $0,046^{a} \pm 0,011$ & $15,16^{\mathrm{a}} \pm 1,87$ & $3,33^{\mathrm{a}} \pm 0,17$ & $54,48^{\mathrm{a}} \pm 2,14$ & $56,41^{\mathrm{a}} \pm 1,13$ & $1942,0^{\mathrm{a}} \pm 277,5$ \\
\hline LMT10 & $11,57^{\mathrm{a}} \pm 2,71$ & $4,10^{\mathrm{a}} \pm 1,08$ & $0,35^{\mathrm{a}} \pm 0,08$ & $0,064^{a} \pm 0,016$ & $15,87^{\mathrm{a}} \pm 1,62$ & $3,34^{\mathrm{a}} \pm 0,22$ & $54,10^{\mathrm{a}} \pm 1,72$ & $59,70^{\mathrm{a}} \pm 1,23$ & $2002,2^{\mathrm{a}} \pm 272,4$ \\
\hline LMT15 & $12,80^{\mathrm{a}} \pm 2,25$ & $4,74^{\mathrm{a}} \pm 0,97$ & $0,33^{\mathrm{a}} \pm 0,06$ & $0,058^{\mathrm{a}} \pm 0,012$ & $15,22^{\mathrm{a}} \pm 1,82$ & $3,21^{\mathrm{a}} \pm 0,20$ & $55,38^{\mathrm{a}} \pm 1,81$ & $56,29^{\mathrm{a}} \pm 2,44$ & $1956,4^{\mathrm{a}} \pm 295,8$ \\
\hline$+\mathrm{N}$ & $6,69^{\mathrm{a}} \pm 1,74$ & $3,03^{\mathrm{a}} \pm 1,33$ & $0,18^{\mathrm{a}} \pm 0,06$ & $0,027 \mathrm{a} \pm 0,008$ & $14,92^{\mathrm{a}} \pm 1,93$ & $3,36^{\mathrm{a}} \pm 0,27$ & $58,33^{\mathrm{a}} \pm 1,34$ & $56,61^{\mathrm{a}} \pm 2,48$ & $2123,3^{\mathrm{a}} \pm 322,8$ \\
\hline \multicolumn{10}{|l|}{ Interacción } \\
\hline $\mathrm{V} * \mathrm{FN}$ & Ns & ns & ns & ns & $*$ & ns & ns & $* * *$ & ns \\
\hline
\end{tabular}

Los valores son promedios de 12 plantas \pm error estándar.

En cada columna, las medias seguidas de la misma letra no difieren estadísticamente (Duncan $\mathrm{p}<0,05$ ).

Duncan significant level: $* 0,05>\mathrm{P}>0,01 ; * * 0,01>\mathrm{P}>0,001 ; * * * \mathrm{P}<0,001$, ns not significant.

VAR: variedad, CCENT: Canario Centenario, CCIF: Canario CIFAC, BM: Blanco Molinero, RM: Rojo Molinero.

-N tratamiento testigo, $+\mathrm{N}$ fertilización nitrogenada, +LMT10 cepa LMT10, LMT15 cepa LMT15.

NN: número de nódulos, TN: tamaño de nódulos, PFN: peso fresco de nódulos, PSN: peso seco de nódulos, NVP: número de vainas por planta, NGV: número de granos por vaina, PCS: peso de cien semillas, IC índice de cosecha, REND: rendimiento.

eso no mostró significancia estadística. Nuestros resultados evidencian que la inoculación con Rhizobium puede reemplazar el uso convencional de fertilizantes que contaminan la atmósfera, debido a que en todas las variedades logramos rendimientos alrededor del $80 \%$ en comparación con lo obtenido con la fertilización nitrogenada e incluso en Blanco y Rojo Molinero se alcanzaron valores similares.

El análisis de los coeficientes de correlación de Pearson entre los datos de rendimiento de las variedades y las variables de la planta mostró el impacto de estos en la variación de rendimiento de cada variedad (Tabla 6). Con base en los resultados obtenidos, el número de vainas por planta y el peso de cien semillas son los parámetros más relacionados con el rendimiento en la variedad Canario Centenario y solo NVP para la variedad Blanco Molinero que presenta el más alto rendimiento $\left(2635.9 \mathrm{~kg} \mathrm{ha}^{-1}\right)$. Tienen más influencia los parámetros sobre el rendimiento de Canario Centenario y Canario CIFAC.

Por otro lado, el análisis de los coeficientes de correlación de Pearson entre los datos de rendimiento de las fuentes de $\mathrm{N}$ y los parámetros de la planta (Tabla 6) muestra que el número de vainas por planta y número de granos por vaina son los parámetros más relacionados significativamente $(\mathrm{p}<0.01)$ con el rendimiento en las fuentes de $\mathrm{N}$, así como los parámetros morfológicos con la fertilización nitrogenada. Además se observa una relación inversa entre los parámetros de nodulación sobre el rendimiento en la cepa LMT10.

\section{Discusiones}

El Perú es considerado un centro de origen y domesticación del frijol (Okii et al., 2014). Excavaciones arqueológicas han mostrado restos de frijol común en la cueva Guitarrero en Perú. Usando el método de datación por radiocarbono, estos restos han sido datados en 7680-10000 a.C. Otros restos incluyen los hallazgos en Nazca o Huaca, hace aproximadamente 2.000 años (Brücher, 1988). En Perú, alrededor del 30\% de los frijoles producidos se cultivan bajo condiciones ecológicas en la zona andina y en la selva (Camarena et al., 2009). Por lo tanto, el uso de cepas de Rhizobium fijadoras de nitrógeno permite obtener rendimientos aceptables en un marco de agricultura sustentable, sin afectar el ecosistema. Las variedades elegidas en el estudio son variedades comerciales ampliamente 
Tabla 6. Coeficientes de correlación lineal de Pearson entre el rendimiento de las variedades y las variables evaluadas en el experimento.

\begin{tabular}{|c|c|c|c|c|c|c|c|c|}
\hline \multirow{2}{*}{ Parameters } & \multicolumn{4}{|c|}{ Variety } & \multicolumn{4}{|c|}{ N Source } \\
\hline & CCENT & CCIF & $\mathrm{BM}$ & $\mathrm{RM}$ & LMT10 & LMT15 & $-\mathrm{N}$ & $+\mathrm{N}$ \\
\hline DTF & 0,065 & $-0,164$ & 0,218 & 0,348 & 0,018 & $0,674^{*}$ & 0,397 & 0,192 \\
\hline ALT & 0,483 & $-0,012$ & $-0,314$ & 0,095 & 0,482 & 0,247 & $0,707 *$ & $0,770 * *$ \\
\hline PFF & 0,328 & 0,073 & $-0,019$ & 0,125 & $0,789 * *$ & 0,493 & 0,550 & $0,629^{*}$ \\
\hline PFR & $-0,166$ & 0,387 & 0,311 & 0,038 & 0,470 & 0,424 & 0,238 & $0,719^{* *}$ \\
\hline PSF & 0,156 & 0,135 & $-0,018$ & 0,148 & $0,729 * *$ & $0,585^{*}$ & $0,576^{*}$ & $0,717 * *$ \\
\hline PSR & $-0,231$ & 0,217 & 0,193 & $-0,087$ & 0,257 & 0,369 & 0,115 & $0,614^{*}$ \\
\hline $\mathrm{NN}$ & $-0,508$ & $-0,136$ & $-0,162$ & $-0,091$ & $-0,128$ & 0,162 & 0,205 & 0,274 \\
\hline $\mathrm{TN}$ & $-0,403$ & $-0,238$ & $-0,343$ & 0,258 & $-0,302$ & $-0,089$ & $-0,139$ & 0,112 \\
\hline PFN & $-0,417$ & $-0,115$ & 0,015 & 0,306 & $-0,167$ & 0,450 & $-0,132$ & 0,304 \\
\hline PSN & $-0,438$ & $-0,115$ & $-0,163$ & 0,306 & $-0,091$ & 0,365 & $-0,128$ & 0,252 \\
\hline NVP & $0,674^{*}$ & 0,419 & $0,687^{*}$ & $0,938 * *$ & $0,822 * *$ & $0,927 * * *$ & $0,921 * * *$ & $0,924 * * *$ \\
\hline NGV & 0,27 & $0,677 * *$ & 0,491 & 0,403 & $0,900 * * *$ & $0,820 * *$ & $0,737 * *$ & $0,812^{* *}$ \\
\hline PCS & $0,720 * *$ & $0,714 * *$ & 0,015 & $-0,428$ & 0,279 & 0,496 & 0,315 & 0,161 \\
\hline IC & 0,17 & $-0,193$ & 0,052 & 0,245 & $-0,271$ & $-0,646^{*}$ & 0,07 & $0,642 *$ \\
\hline
\end{tabular}

Nivel de significación de Pearson: *0,05 > P > 0,01; **0,01 > P > 0,001; ***P $<0,001$.

VAR: variedad, CCENT: Canario Centenario, CCIF Canario CIFAC, BM: Blanco Molinero, RM: Rojo Molinero, DAF: días a la floración, ALT: altura, PFF: peso fresco de follaje, PFR: peso fresco de raíces, PSF: peso seco de follaje, PSR: peso seco de raíces, NN: número de nódulos, TN: tamaño de nódulos, PFN: peso fresco de nódulos, PSN: peso seco de nódulos, NVP: número de vainas por planta, NGV: número de granos por vaina, PCS: peso de cien semillas, IC: índice de cosecha, REND: rendimiento.

consumidas en el Perú. Cada variedad se clasifica por su color (Camarena et al., 2009) y según este criterio, las preferencias de consumo varían entre las regiones del Perú. Aproximadamente el 60\% de la producción es consumida en la costa central. En esta zona es determinante la preferencia por el grano de color amarillo que corresponde a la clase comercial Canario (Voysest, 2000).

En este estudio seleccionamos dos cepas de Rhizobium aisladas de la variedad Blanco Molinero para determinar la correlación entre las cepas y las variedades sobre el crecimiento, nodulación y rendimiento del frijol, además de observar las posibles diferencias en la nodulación según el color de la variedad (Tabla 7).

En términos de características cualitativas, casi todos los tratamientos tienen nódulos en las raíces laterales, en la zona media, porque allí las raíces están en constante crecimiento activo y los nódulos se forman en ellas. Los nódulos son predominantemente esféricos, pero en algunas variedades estaba presente la forma elíptica (Tabla 7). Según Newcomb, citado por Nap \& Bisseling (1990), esta forma se encuentra en los géneros Phaseolus, Glycine, Vigna y Lotus. El color interno del nódulo varía de rosado y rojo a marrón rojizo (Tabla 7). Aunque el color rojo y rosado está asociado con la leghemoglobina y la fijación activa de nitrógeno, otros autores han encontrado que las cepas ineficaces pueden tener nódulos rojos, incluso negros (Unkovich et al., 2008).

En el Perú, muy pocos experimentos han sido realizados bajo condiciones de campo con diversas variedades. Generalmente se han hecho en una sola variedad (Manrique et al., 1993; Camarena et al., 2009). Estos estudios previos muestran una respuesta contradictoria de la nodulación y el efecto de la simbiosis sobre el crecimiento, desarrollo y productividad del frijol (Bliss y Hardarson, 1993). Los datos obtenidos demuestran un incremento considerable en el peso fresco y seco de raíces en todas las variedades, excepto la Rojo Molinero que obtuvo valores bajos en todos los parámetros evaluados debido a que tiene requerimientos de temperaturas más altas para un óptimo desarrollo. Los elevados rendimientos con los tratamientos inoculados respecto al control, aunque sin diferencias significativas para las fuentes de nitrógeno, evidencian la alta competitividad y la eficiencia de las cepas de Rhizobium comparado con los tratamientos fertilizados. La presencia de nódulos en tratamientos fertilizados puede ser por la aplicación de cobalto a través de Oligomix Co, disminuyendo los efectos inhibitorios del nitrato en la nodulación (Jain y Nainawatee, 2000). El alto 
Tabla 7. Características de nodulación para los tratamientos.

\begin{tabular}{|c|c|c|c|c|c|c|c|c|c|c|}
\hline Variedad & Fuente de N & $\mathrm{T}$ & $\begin{array}{c}\text { Peso Fresco } \\
\text { de Nódulos } \\
\text { (G) }\end{array}$ & $\begin{array}{l}\text { Peso Seco } \\
\text { de Nódulos } \\
(\mathrm{G})\end{array}$ & $\begin{array}{l}\text { Número } \\
\text { de } \\
\text { Nódulos }\end{array}$ & $\begin{array}{l}\text { Tamaño de } \\
\text { Nódulos } \\
(\mathrm{Cm})\end{array}$ & $\begin{array}{l}\text { Posición } \\
\text { de los } \\
\text { Nódulos }\end{array}$ & $\begin{array}{c}\text { Forma } \\
\text { de los } \\
\text { Nódulos }\end{array}$ & $\begin{array}{c}\text { Color } \\
\text { Interno del } \\
\text { Nódulo }\end{array}$ & $\begin{array}{c}\% \\
\text { Nódulos } \\
\text { Efectivos }\end{array}$ \\
\hline & Cepa LMT10 & $\mathrm{T} 1$ & 0,43 & 0,06 & 15,33 & 3,56 & Medio & Esférico & Marrón rojizo & 70 \\
\hline Canario & Cepa LMT15 & $\mathrm{T} 2$ & 0,45 & 0,06 & 11,14 & 4,06 & Medio-inferior & Esférico & Rojo & 80 \\
\hline \multirow[t]{3}{*}{ Centenario } & Cepa nativa & $\mathrm{T} 3$ & 0,24 & 0,03 & 8,79 & 5,59 & Superior-medio & Elíptico & Rosado & 60 \\
\hline & Fertilización N & $\mathrm{T} 4$ & 0,13 & 0,02 & 5,89 & 2,25 & Medio & Esférico & Rojo & 20 \\
\hline & Cepa LMT10 & $\mathrm{T} 5$ & 0,12 & 0,02 & 7,49 & 6,88 & Medio & Elíptico & Rosado & 80 \\
\hline Canario & Cepa LMT15 & $\mathrm{T} 6$ & 0,20 & 0,03 & 12,50 & 8,75 & Medio & Esférico & Rojo & 60 \\
\hline \multirow[t]{3}{*}{ CIFAC } & Cepa nativa & $\mathrm{T} 7$ & 0,11 & 0,02 & 8,39 & 6,37 & Medio-inferior & Esférico & Rosado & 70 \\
\hline & Fertilización N & $\mathrm{T} 8$ & 0,10 & 0,02 & 5,56 & 6,14 & Todo & Esférico & Rosado & 30 \\
\hline & Cepa LMT10 & T9 & 0,49 & 0,11 & 14,93 & 1,83 & Medio-inferior & Esférico & Rojo & 80 \\
\hline Blanco & Cepa LMT15 & $\mathrm{T} 10$ & 0,47 & 0,10 & 17,03 & 2,23 & Medio & Esférico & Marrón rojizo & 70 \\
\hline \multirow[t]{3}{*}{ Molinero } & Cepa nativa & T11 & 0,44 & 0,07 & 16,65 & 2,11 & Medio-inferior & Elíptico & Marrón rojizo & 50 \\
\hline & Fertilización N & $\mathrm{T} 12$ & 0,43 & 0,06 & 13,48 & 2,13 & Medio-inferior & Esférico & Rosado & 30 \\
\hline & Cepa LMT10 & $\mathrm{T} 13$ & 0,36 & 0,06 & 8,54 & 4,13 & Todo & Elíptico & Marrón rojizo & 90 \\
\hline Rojo & Cepa LMT15 & $\mathrm{T} 14$ & 0,20 & 0,03 & 10,54 & 3,92 & Superior-medio & Elíptico & Marrón rojizo & 85 \\
\hline \multirow{2}{*}{ Molinero } & Cepa nativa & $\mathrm{T} 15$ & 0,41 & 0,07 & 11,72 & 6,12 & Superior-medio & Esférico & Rojo & 60 \\
\hline & Fertilización N & T16 & 0,05 & 0,01 & 1,83 & 1,58 & Todo & Esférico & Rosado & 30 \\
\hline Promedio & & & 0,29 & 0,05 & 10,61 & 4,23 & - & - & - & 60 \\
\hline
\end{tabular}

número de vainas por planta en Canario Centenario se debe a que las condiciones climáticas fueron perfectas para esta variedad, con temperaturas moderadas a altas en el día y bajas en la noche, lo que produce una mayor cantidad de fotosintatos en el día que pueden ser translocados para el llenado de los granos y vainas (Parker, 2004). Basados en nuestros resultados, determinamos que el efecto de la inoculación de las cepas fue dependiente de las variedades, lo que coincide con lo reportado por Sánchez et al. (2014).

La inoculación de las leguminosas, especialmente de frijol común, tiene un gran potencial para incrementar los rendimientos con menores costos de producción que usando fertilizantes químicos. Por lo tanto es ventajoso seleccionar cepas efectivas para variedades específicas de la región donde se cultivan los frijoles. Nuestros resultados muestran una actividad superior de la combinación de la cepa LMT15 en la Blanco Molinero, debido a que fue aislada de esta variedad. El uso de inoculación con cepas nativas puede ofrecer una ventaja significativa para el éxito en la nodulación y el rendimiento de las leguminosas cultivadas, como se demuestra en nuestro trabajo. La variedad Blanco Molinero con cepas nativas obtiene un rendimiento de $2.836 \mathrm{~kg}$ $\mathrm{ha}^{-1}$ que es alto en comparación con lo logrado hoy en día con fertilizaciones intensivas en nuestro país, que tiene un promedio de $2.000 \mathrm{~kg} \mathrm{ha}^{-1}$ (Camarena et al., 2009; Mulas et al., 2011).

\section{Conclusiones}

En conclusión, todas las variedades inoculadas con Rhizobium producen mayores rendimientos y número de nódulos que aquellas que no han sido inoculadas o que han sido fertilizadas con nitrógeno. Ello demuestra el potencial de la inoculación para mejorar la productividad del frijol común. En algunos casos, no se encontraron diferencias, como en la variedad Rojo Molinero, debido probablemente a su cultivo en condiciones ambientales no favorables. Sin embargo, los beneficios en el rendimiento del frijol han sido observados con las cepas inoculadas. Por lo tanto, estos datos pueden ser corroborados en pruebas de campo y de aplicación comercial a gran escala en el país.

\section{Agradecimientos}

Este trabajo fue parcialmente subvencionado por el Vicerrectorado Académico de la UNALM, con mención especial al Dr. Jorge Aliaga Gutiérrez y a la Ing. Diana Castro Álvarez. Se agradece también a los miembros del Programa de Investigación y Proyección Social en Leguminosas y Oleaginosas, por su orientación en la culminación del estudio. 


\section{Literatura Citada}

Abayomi, Y.A.; Ajibade, T.V.; Sammuel, O.F.; Sáadudeen, B.F. 2008. Growth and yield responses of cowpea (Vigna unguiculata (L.) Walp) genotypes to nitrogen fertilizer (NPK) application in the Southern Guinea Savanna zone of Nigeria. Asian J. Plant Sci, 7 (2): 170-176.

Alberton, O.; Kaschuk, G.; Hungria, M.

2006. Sampling effects on the assessment of genetic diversity of rhizobia associated with soybean and common bean. Soil Biology and Biochemistry, 38: 1298-1307.

Rahmani, H.A.; Räsänen, LA.; Afshari M.; Lindström, K.

2011. Genetic diversity and symbiotic effectiveness of rhizobia isolated from root nodules of Phaseolus vulgaris L. grown in soils of Iran. Applied Soil Ecology, 48 (2011): 287-293.

Bliss, F.A. \& Hardarson, G.

1993. Enhancement of Biological Nitrogen Fixation of Common Bean in Latin America. Plant Soil, 152, 1-160.

Broughton, W.J.; Hernández, G.; Blair, M.; Beebe, S.; Gepts, P.; Vanderleyden J.

2003. Beans (Phaseolus spp.)- model food legumes. Plant and Soil, 252 (1): 55-128.

Brücher, $\mathrm{H}$.

1988. The wild ancestor of Phaseolus vulgaris in South America. En: Gepts, P. (ed.). Genetic Resources of Phaseolus Beans, 185-214. Kluwer Academic Publishers. Dordrecht, The Netherlands. pp. 185-214.

Camarena, M.F.; Huaringa, J.A.; Mostacero, N.E.

2009. Innovación Tecnológica para el incremento de la producción de frijol común (Phaseolus vulgaris L.). Primera Edición. Universidad Nacional Agraria La Molina - Consejo Nacional de Ciencia, Tecnología e Innovación Tecnológica. Lima, Perú. 232 p.

Erisman, J.W.

2011. The New Global Nitrogen Cycle. En: Polacco, J.C.; Todd, D. 2011. Ecological Aspects of Nitrogen Metabolism in Plants. First Edition. John Wiley \& Sons, Inc. N.J., U.S. pp. 5-15

Jain, V.; Nainawatee, H.S.

2000. Cobalt reduces nitrate inhibition of nodulation in mungbean (Vigna radiata L.). Biology and Fertility of Soils, 31 (6): 522-524.

Kwak, M.; Gepts, P.

2009. Structure of genetic diversity in the two major gene pools of common bean (Phaseolus vulgaris L., Fabaceae). Theoretical and Applied Genetics, 118 (5): 979-992.

Lichtfouse, E.; Navarrete, M.; Debaeke, P.; Souchére, V.; Alberola, C. \& Ménassieu, J.

2008. Agronomy for sustainable agriculture. A review. Agron. Sustain. Dev., 29: 1-7.

Manrique, A.; Manrique, K.; Nakahodo, J.

1993. Yield and biological nitrogen fixation of common bean (Phaseolus vulgaris L.) in Peru. Plant and Soil, 152: 87-91.

Mathu, S.; Herrmann, L.; Pypers, P.; Matiru, V.; Mwirichia,

R, Lesueur, D.

2012. Potential of indigenous Bradyrhizobia versus commercial inoculants to improve cowpea (Vigna unguiculata L. Walp.) and green gram (Vigna radiata L. Wilczek.) yields in Kenya. Soil Science and Plant Nutrition, 58(6): 750-763.
Mulas, D.; García-Fraile, P.; Carro, L.; Ramírez-Bahena, M.H.; Casquero, P.; Velázquez, E.; Gonzales-Andrés, F.

2011. Distribution and efficiency of Rhizobium leguminosarum strains nodulating Phaseolus vulgaris in Northern Spanish soils: Selection of native strains that replace conventional N fertilization. Soil Biology and Biochemistry, 43 (11): 2283-2293.

Nap, J.P.; Bisselling, T.

1990. Developmental biology of a plant prokaryote symbiosis: The legume root nodule. Science, 250 (4983): 948-954.

Ndungu, S.M.; Messmer, M.M.; Ziegler, D.; Thuita, M.; Vanlauwe, B.; Frossard, E.; Thonar, C.

2018. Evaluation of MALDI-TOF mass spectrometry for the competitiveness analysis of selected indigenous cowpea (Vigna unguiculata L. Walp.) Bradyrhizobium strains from Kenya. Applied Microbiology and Biotechnology, 102 (12): 5265-5278.

Nótás, E.; Debreczeni, K.; Berecz, K.; Heltai, G.

2007. Effect of $\mathrm{N}$ fertilizers and soil moisture on the $\mathrm{N}$-gaseous losses and the plant $\mathrm{N}$ uptake in a maize pot experiment. Cereal Research Communications, 35 (2): 853-856.

Okii, D.; Tukamuhabwa, P.; Kami, J.; Namayanja, A.; Paparu, P.; Ugen, M.; Gepts, P.

2014. The genetic diversity and population structure of common bean (Phaseolus vulgaris L) germplasm in Uganda. African Journal of Biotechnology, 13 (29): 2935-2949.

Parker, Rick.

2004. Introduction to Plant Science, Delmar Learning. 736 p.

Pedrosa, M.M.; Cuadrado, C.; Burbano, C.; Muzquiz, M.;

Cabellos, B.; Olmedilla-Alonso, B.; Asensio-Vegas, C.

2015. Effects of industrial canning on the proximate composition, bioactive compounds contents and nutritional profile of two Spanish common dry beans (Phaseolus vulgaris L.). Food Chemistry, 166: 68-75.

Rodríguez-Navarro, D.N.; Buendía, A.M.; Camacho, M.; Lucas, M.; Santamaría, C.

2000. Characterization of Rhizobium spp. bean isolates from southwest of Spain. Soil Biology and Biochemistry, 32: 1601-1613.

Sánchez, A.C.; Gutiérrez, R.T.; Santana, R.C.; Urrutia, A.R.; Fauvart, M.; Michiels, J.; Vanderleyden, J.

2014. Effects of co-inoculation of native Rhizobium and Pseudomonas strains on growth parameters and yield of two contrasting Phaseolus vulgaris L. genotypes under Cuban soil conditions. European Journal of Soil Biology, 62: 105-112.

Soil Survey Staff.

2014. Soil Survey Field and Laboratory Methods Manual. Soil Survey Investigations Report No. 51, Version 2.0. R. Burt and Soil Survey Staff (ed.). Department of Agriculture, Natural Resources Conservation Service. U.S.A. 457 p.

Unkovich, M.; Herridge, D.; Peoples M.; Cadisch, G.; Boddey, B.; Giller, K.; Alves, B. \& Chalk, P.

2008. Measuring plant-associated nitrogen fixation in agricultural systems. Australian Centre for International Agricultural Research Monograph No 136. Canberra, Australia. 258 p.

Voysest, O.

2000. Mejoramiento Genético del Frijol (Phaseolus vulgaris L.): Legado de Variedades de América Latina 1930-1999. Centro Internacional de Agricultura Tropical (CIAT). Cali, Colombia. $195 \mathrm{p}$. 
